\title{
The Active Target Time Projection Chamber at NSCL
}

\author{
D. Bazin ${ }^{1,2, \star}$, J. Bradt ${ }^{1,2}$, Y. Ayyad ${ }^{3}$, W. Mittig ${ }^{1,2}$, T. Ahn ${ }^{4}$, S. Beceiro-Novo ${ }^{1,2}$, L. Carpenter ${ }^{1,2}$, M. Cortesi ${ }^{1}$, A. Fritsch ${ }^{5}$, \\ J. J. Kolata ${ }^{4}$, W. Lynch ${ }^{1,2}$, and N. Watwood ${ }^{1,6}$ \\ ${ }^{1}$ National Superconducting Cyclotron Laboratory, Michigan State University, East Lansing, MI 48824-1321, USA \\ ${ }^{2}$ Department of Physics and Astronomy, Michigan State University, East Lansing, MI 48824-1321, USA \\ ${ }^{3}$ Department of Applied Nuclear Physics, Lawrence Berkeley National Laboratory, Berkeley, CA 94720, USA \\ ${ }^{4}$ Department of Physics, University of Notre Dame, Notre Dame, IN 46556, USA \\ ${ }^{5}$ Department of Physics, Gonzaga University, Spokane, WA 99258, USA \\ ${ }^{6}$ Department of Chemistry, Michigan State University, East Lansing, MI 48824-1321, USA
}

\begin{abstract}
Reactions in inverse kinematics close to the Coulomb barrier offer unique opportunities to study exotic nuclei, but they are plagued by the difficulty to efficiently and precisely measure the characteristics of the emerging particles. The Active Target Time Projection Chamber (AT-TPC) offers an elegant solution to this dilemma. In this device, the detector gas of the time projection chamber is at the same time the target in which nuclear reactions take place. The use of this new paradigm offers several advantages over conventional inert target methods, the most significant being the ability to increase the luminosity of experiments without loss of resolution. The AT-TPC and some results obtained on resonant $\alpha$ scattering to explore the clustering properties of neutron-rich nuclei are presented, as well as fusion cross section results using a ${ }^{10} \mathrm{Be}$ radioactive beam. In addition, the first re-accelerated radioactive beam experiment using the fully commissioned ReA3 linac was conducted recently at the NSCL with the AT-TPC, where proton resonant scattering of a $4.6 \mathrm{MeV} / \mathrm{u}^{46} \mathrm{Ar}$ beam was used to measure the neutron single-particle strength in ${ }^{47} \mathrm{Ar}$.
\end{abstract}

\section{Introduction}

Reactions close to the Coulomb barrier have been used with light beam probes such as protons, deuterons or alphas since the dawn of the first accelerators. The properties of the majority of stable isotopes found in the chart of the nuclides have been studied using a wide variety of reactions such as resonant scattering or transfer reactions. However, extending these techniques to radioactive nuclei produced as beams has proven to be a significant experimental challenge. The main reason lies with inverse kinematics where the light probe nuclei are at rest in a target and have much wider kinetic energy range after the reaction than in direct kinematics. Measuring the properties of the scattered probe or its remnant becomes much more challenging, and in most cases a compromise between target thickness and resolution becomes necessary.

The concept of active target is an attempt at avoiding this compromise by turning the target into a detector medium where all the energy dissipated during the reaction can be measured and the location of each reaction vertex determined experimentally [1]. This is best done in a gas volume where the radioactive beam can react with the gas atomic nuclei as it slows down. Since the energy of the reaction products can be measured as they emerge from the reaction vertex, there is no loss of resolution regardless of the amount of material traversed by the incoming beam.

\footnotetext{
$\star^{\star}$ e-mail: bazin @nscl.msu.edu
}

The gas pressure can be adjusted to vary the effective target thickness and measure reactions in a particular range of energies. From the reaction vertex location measurement, the energy of the beam at the time of the reaction can be determined for each event and an excitation function of the cross section can be deduced. This feature is particularly useful when studying reactions such as fusion or resonant scattering for instance.

The AT-TPC and its smaller scale prototype (pATTPC) have been used at NSCL and other laboratories to conduct these types of experiments at energies close to the Coulomb barrier. Most of the experiments conducted so far have used low energy resonant scattering and fusion reactions. A few examples of the results obtained are presented in the following, after a brief description of the detector and its associated technology.

\section{Detector}

The AT-TPC consists of a 250 liter cylindrical volume closed by a cathode plate at the upstream end, and a sensor plane (labeled Micromegas) at the downstream end (see fig. 1). Upon applying a voltage at the cathode end, electrons freed by charged particles along their track drift towards the sensor plane at a constant velocity. The electric field is kept uniform along the main axis of the cylinder by a series of concentric rings located both inside and outside the field cage, and polarized via a linear resistor 
chain. Upon arriving at the sensor plane, the electrons

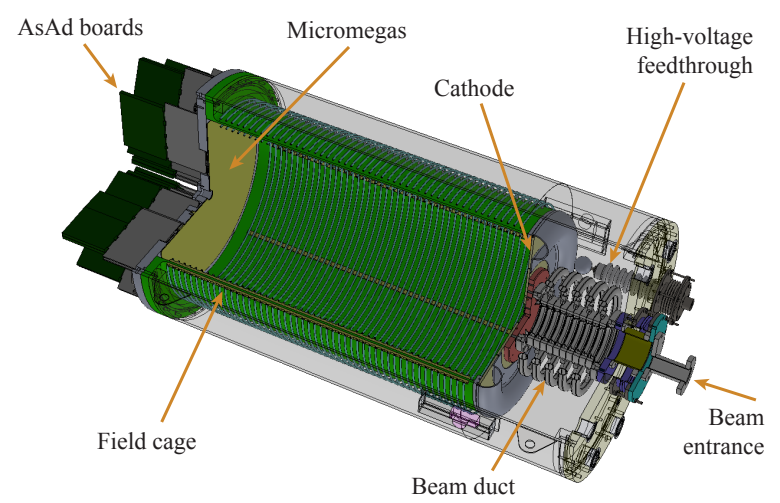

Figure 1. Schematic view of the AT-TPC. The field cage is made of a non-conductive material, fiberglass and epoxy. The outer chamber shields the field cage from the exterior and holds the beam duct that couples the high voltage of the cathode to the grounded beam pipe. The beam enters the field cage through a small $3 \mu \mathrm{m}$-thick window placed at the center of the cathode. The front-end cards (AsAd boards) of the digital electronics are directly connected to the back of the sensor plane via small feedthrough boards equipped with o-rings for sealing the inner volume to the exterior.

are multiplied by a Micromegas device [2] consisting of a micro mesh mounted on a pad plane made of a Printed Circuit Board (PCB) where a pattern of 10,240 triangular pads have been etched. On the other side of this PCB high density connectors are used to transfer the signals to the digital electronics. A 3-dimensional image of the tracks left by the charged particles inside the field cage can be reconstructed from a time recording of the signals on each pad. The AT-TPC can be placed inside a large bore MRI solenoid to apply a magnetic field superimposed on the electric field. The resulting curved trajectories can be analyzed to measure the magnetic rigidity of the emitted particles for identification as well as momentum determination. The prototype version of the AT-TPC was built first and is very similar, except for its size, the geometry of the sensor plane and the number of channels [3].

The high density electronics used with the AT-TPC has been specially developed by a collaboration (GET for General Electronics for TPC) [4], that features for each channel a charge preamplifier and shaping amplifier followed by a 512-cell analog memory and 12-bit ADC for the digitizing section. In addition, this electronics has the unique feature of implementing discriminators on each channel and combining them into a running multiplicity that can be used to generate an internal trigger. This feature is particularly important in an active target as all charged particles entering the field cage do not escape, therefore no external detector can be used to generate a trigger from them. A complete description of the full scale AT-TPC will be available in a forthcoming publication [5].

\section{Results}

A few results obtained with the AT-TPC detectors are presented. They involve reactions around the Coulomb barrier which is the domain most adapted to the active target concept.

\section{$3.1 \alpha$ resonant scattering}

$\alpha$ resonant scattering at low energy is an efficient reaction to populate virtual states (also referred to as resonances) of the target+projectile composite system. In the case of $\alpha$ particles, these resonances may exhibit $\alpha$ clustering effects that are of high interest in the nuclear physics community. The most famous $\alpha$ cluster state is the so-called Hoyle state of ${ }^{12} \mathrm{C}$, that is critical in the description of ${ }^{12} \mathrm{C}$ nucleo-synthesis via the triple $\alpha$ reaction. $\alpha$ cluster states are sought in other light nuclei, that may have equally important impacts on nucleo-synthesis, but are also of great interest in structure models of the nucleus.

The $\alpha$ clustering resonances in the nuclei ${ }^{10} \mathrm{Be}[6],{ }^{14} \mathrm{C}$ [7] and more recently ${ }^{12} \mathrm{Be}$ have been investigated with the pAT-TPC using radioactive beams of ${ }^{6} \mathrm{He},{ }^{10} \mathrm{Be}$ and ${ }^{8} \mathrm{He}$ respectively. The target was composed of ${ }^{4} \mathrm{He}$ gas mixed with a $10 \%$ concentration of $\mathrm{CO}_{2}$ as quencher. The radioactive beams were produced at the Notre Dame tandem facility using the Twinsol device [8] for [6, 7], and the TRIUMF facility for the ${ }^{8} \mathrm{He}$ beam.

The results shown below were obtained with the ${ }^{10} \mathrm{Be}$ beam, and show a clear separation between the elastic scattering events and the inelastic events where ${ }^{10} \mathrm{Be}$ was excited to its first $2^{+}$state (panel b of fig. 2). The tracks from ${ }^{4} \mathrm{He}$ and ${ }^{10} \mathrm{Be}$ were clearly identified from the respective energy losses of the two particles, helped by polarizing every $5^{\text {th }}$ ring of the pad plane to a higher gain for the Micromegas [7], as seen on panel a of the same figure 2. From the track reconstruction, the vertex of the reactions
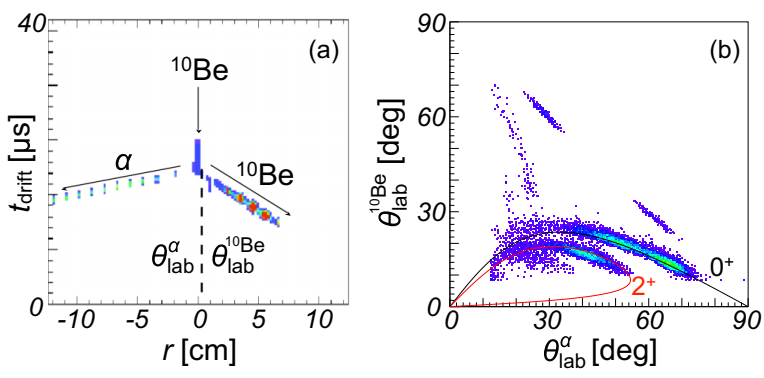

Figure 2. Panel a: typical scattering event recorded in the pATTPC, showing the double-gain setup of the Micromegas, by polarizing every $5^{\text {th }}$ ring of the pad plane to a higher gain in order to better cover the dynamic range differences between the ${ }^{4} \mathrm{He}$ and ${ }^{10} \mathrm{Be}$ particles. Panel b: kinematics plot of the angle of ${ }^{10} \mathrm{Be}$ vs the angle of ${ }^{4} \mathrm{He}$, where the elastic and inelastic components of the cross section are clearly separated (from [7]).

can be determined event by event, then used to calculate the reaction energy. Excitation functions can then be deduced as a function of the center-of-mass angle, as shown in panels a and $c$ of fig. 3. The excitation functions show a 


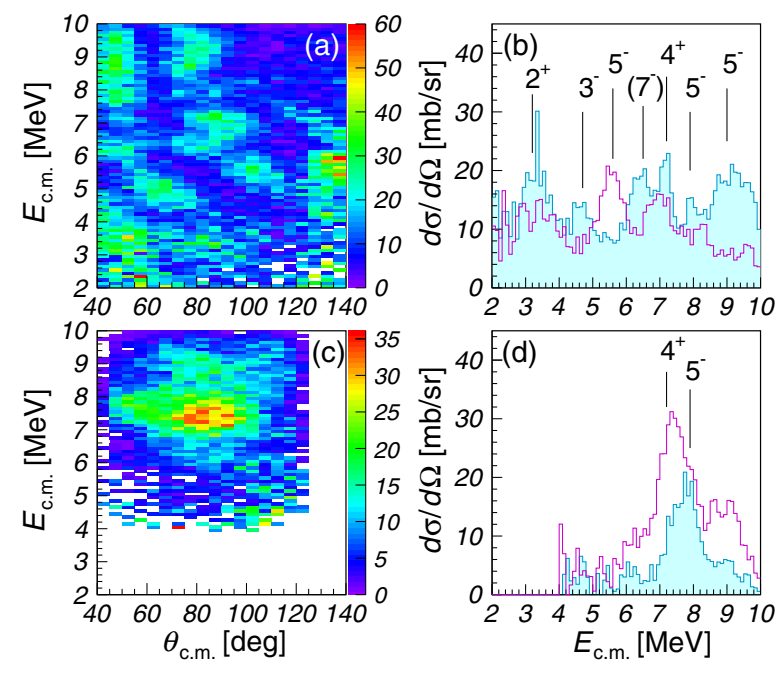

Figure 3. Panels a and c: excitation functions plotted for different center-of-mass angle of the reaction for the elastic and inelastic components of the cross section, respectively. The projections of the excitation functions between angles $70^{\circ}-90^{\circ}$ (shaded spectrum) and $90^{\circ}-110^{\circ}$ (clear spectrum) (from [7]).

number of resonances which can be identified via R-matrix analysis of their angular distributions. In this work $2^{+}$and $4^{+}$resonances were identified at $3.0 \mathrm{MeV}$ and $7.0 \mathrm{MeV}$, respectively and tentatively assigned to a rotational band corresponding to a predicted linear chain structure in ${ }^{14} \mathrm{C}$ [9]. The predicted $0^{+}$band head resonance was not observed likely due to the $2 \mathrm{MeV}$ experimental trigger cutoff.

\subsection{Proton resonant scattering}

Proton resonant scattering has been used extensively using proton beams to extract spectroscopic information on the analog states of the populated resonances in the neutron plus target system of the same isospin. The idea is to populate $T_{0}+\frac{1}{2}$ compound nucleus states of a $T_{0}{ }^{A} Z$ nucleus plus a proton that are isospin analogs of bound levels in the ${ }^{A+1} Z$ nucleus. An example can be found in [10] for the case of ${ }^{41} \mathrm{Ar}$ where the authors find good agreement between their deduced spectroscopic factors and those obtained from a ${ }^{40} \mathrm{Ar}(\mathrm{d}, \mathrm{p})$ transfer reaction. The $\mathrm{T}_{>}$resonances observed in [10] were identified and analyzed by averaging the excitation functions with a width of about $20 \mathrm{keV}$, in order to smooth out the superimposed $\mathrm{T}_{<}$resonances of the ${ }^{40} \mathrm{~K}$ compound nucleus.

Although many stable isotopes have been studied via this method using intense proton beams of very well defined energies, only a few radioactive isotopes with long enough half-lives to make target material could be measured. Carrying this methodology to short-lived radioactive nuclei implies the use of radioactive beams, therefore inverse kinematics. The motivation for measuring proton resonant scattering on ${ }^{46} \mathrm{Ar}$ stems from the observation of the weakening of the $\mathrm{N}=28$ shell gap in isotones of ${ }^{48} \mathrm{Ca}$ such as ${ }^{44} \mathrm{~S}$ and ${ }^{42} \mathrm{Si}[11,12]$. Located between the doubly magic ${ }^{48} \mathrm{Ca}$ and highly deformed ${ }^{44} \mathrm{~S},{ }^{46} \mathrm{Ar}$ could exhibit reduced single-particle strength [13], and impose meaningful constraints on the effective interactions used in shell model calculations. Investigations using Coulomb excitation [14] and transfer reactions [15] indicate such a reduction might be small, and using an orthogonal method such as proton resonant scattering offers an alternative way of measuring spectroscopic factors in ${ }^{47} \mathrm{Ar}$.

Performing this type of measurement in inverse kinematics on radioactive beams requires good quality beam properties. The energy resolution in particular is critical because the reaction energy is deduced from the position of the reaction vertex and the corresponding energy loss of the beam. The re-accelerated ${ }^{46} \mathrm{Ar}$ beam used to perform proton resonant scattering on this nucleus had an energy of $4.6 \mathrm{MeV} / \mathrm{u}$ with an energy width below $1 \%$, hence an energy resolution around $40 \mathrm{keV} / \mathrm{u}$. This radioactive beam was first produced via projectile fragmentation of a 140 $\mathrm{MeV} / \mathrm{u}^{48} \mathrm{Ca}$ primary beam on a ${ }^{9} \mathrm{Be}$ target, filtered by the A1900 fragment separator, then stopped in a gas cell before being injected into an electron beam charge breeder, accelerated by the ReA3 linac and delivered to the ATTPC. The average beam intensity was around 1,500 particles per second, with a small admixture (around 10\%) of the stable ${ }^{60} \mathrm{Ni}$ and a few $\%$ of the ${ }^{46} \mathrm{Ar}$ daughter and grand-daughter ${ }^{46} \mathrm{~K}$ and ${ }^{46} \mathrm{Ca}$. A small ion chamber was placed in front of the AT-TPC to measure the energy loss of the incoming particles for identification. Fig. 5 shows the vertex position distribution observed in this experiment, where the sharp falloff corresponds to the entrance window of the field cage. The position resolution obtained from fitting the falloff edge of the distribution is $14.1 \mathrm{~mm}$ FWHM, which corresponds to an energy resolution of 43.3 $\mathrm{keV} / \mathrm{u}$ FWHM.

Several challenges were encountered during this experiment. In particular, the large difference in energy loss between the ${ }^{46} \mathrm{Ar}$ and proton caused saturation and distortion effects in the electronics that required extensive cleaning of the data during the analysis phase. Unlike in the ${ }^{10} \mathrm{Be}+{ }^{4} \mathrm{He}$ experiment presented above, individual polarization of the 10,240 pads of the AT-TPC was not available to reduce the avalanche gain of the pads on which the ${ }^{46} \mathrm{Ar}$ beam tracks were projected. This shortcoming is now being addressed via electronically controlled protection boards that allow the polarization of individual pads to be set programmatically.

The proton scattering event displayed in fig. 4 has been processed using image recognition algorithms such as the Hough transform to remove noise and spurious data. The left-hand side shows the pad plane projection of the event, while the right-hand side shows projections of the same event together with the result of the Monte-Carlo fitting used to model the event and extract its physical parameters such as vertex energy, scattering and azimuthal angles. More details on the analysis method can be found in [5]. The gas target used in this experiment was $\mathrm{C}_{4} \mathrm{H}_{10}$ at about 20 Torr, therefore events corresponding to scattering on ${ }^{12} \mathrm{C}$ nuclei had to be rejected from the analysis. The final phase of this complex analysis is still ongoing and will be the subject of a publication in the near future. 


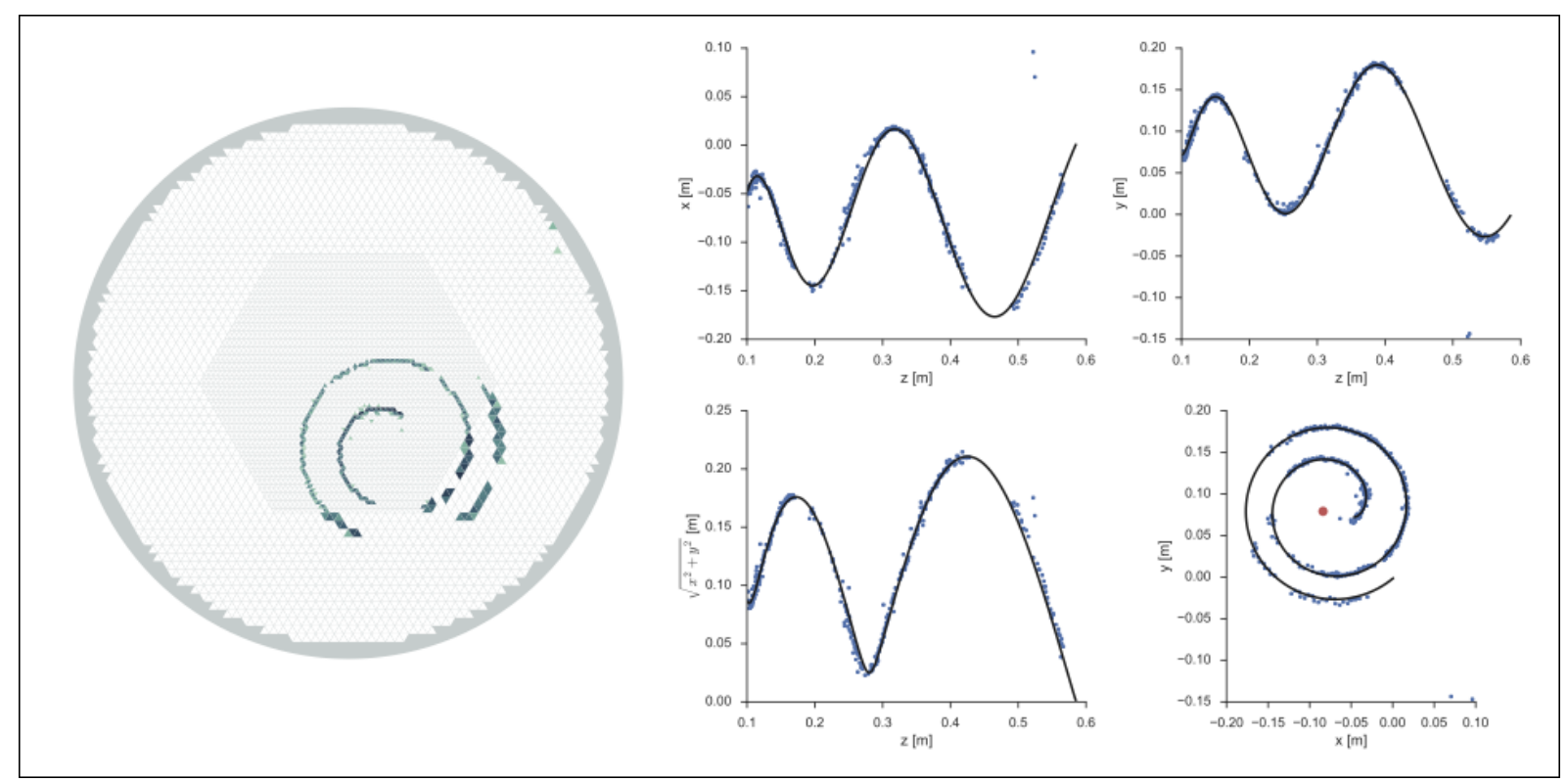

Figure 4. Typical event observed in ${ }^{46} \mathrm{Ar}+\mathrm{p}$ scattering. The spiral track corresponds to the recoil proton orbiting in the 1.7 Tesla solenoidal field and slowing down in the gas. The hit pattern (left) and projections (right) show the data after processing designed to remove noise and cross talk, resulting in significant fragmentation of the track. The right-hand side projections have the results of the Monte-Carlo fit superimposed on the data. The red dot indicates the center of the spiral as guessed from the initial circular Hough transformation algorithm.

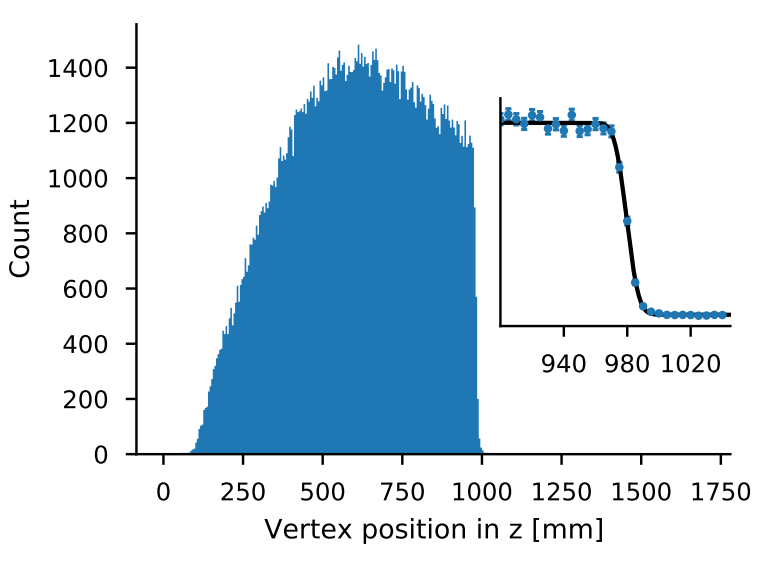

Figure 5. Vertex position distribution measured for the ${ }^{46} \mathrm{Ar}+\mathrm{p}$ reaction. The sharp falloff corresponds to the entrance window of the field cage. The inset shows the detail of the falloff and the fit function used to estimate the resolution.

\subsection{Fusion}

For the same reasons as explained in the previous sections, the AT-TPC is particularly well adapted to measure fusion cross section excitation functions. The following results were obtained using a ${ }^{10} \mathrm{Be}$ radioactive beam produced by the Twinsol device at the University of Notre Dame [16]. Using P10 gas (90\% Ar and $\left.10 \% \mathrm{CH}_{4}\right)$ in the pATTPC, the fusion cross section between ${ }^{10} \mathrm{Be}$ and ${ }^{40} \mathrm{Ar}$ was measured with very low beam intensity (100 counts per second). The selection and identification of fusion events was achieved using the range and energy loss information

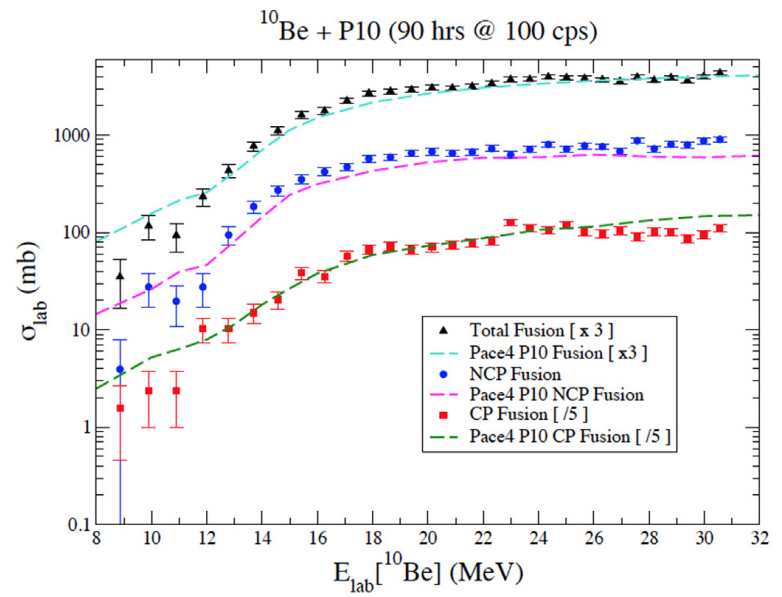

Figure 6. Excitation function of the ${ }^{40} \mathrm{Ar}+{ }^{10} \mathrm{Be}$ fusion cross section. The total fusion cross section, as well as fusion cross section where a charged particle was emitted (CP) or not (NCP) are compared to Pace4 calculations (from [16]).

recorded by the detector. The observation of events where a charged particle was emitted during the reaction enabled us to separate fusion cross sections in different categories, as shown in fig. 6 .

\section{Conclusion}

The active target concept combined with a time projection chamber detector shows promising prospects for experiments using rare radioactive beams in inverse kinematics at energies close to the Coulomb barrier. Several experi- 
ments have already shown interesting results in the study of $\alpha$ clustering states of neutron-rich nuclei and fusion cross section measurements. Resonant proton scattering turns out to be more challenging experimentally, in particular due to the large size difference between the beam and recoil particle, but the first experiment conducted with a re-accelerated beam of ${ }^{46} \mathrm{Ar}$ from the ReA3 linac at NSCL shows the potential of this method for exploring the single-particle structure of nuclei far from stability. Several improvements aimed at mitigating the shortcomings observed during this commissioning run are well under way, and will greatly benefit future experiments.

\section{Acknowledgements}

This work is supported by the US National Science Foundation under Grants No. MRI09-23087, No. PHY0969456 and No. PHY14-01343.

\section{References}

[1] S. Beceiro-Novo et al., Prog. in Part. and Nucl. Phys. 84, 124 (2015).
[2] Y. Giomataris et al., Nucl. Instrum. Methods Phys. Res. A 376, 29 (1996).

[3] D. Suzuki et al., Nucl. Instrum. and meth. in Phys. Res. A 691, 39 (2012).

[4] E. Pollacco et al., Physics Procedia 37, 1799 (2012).

[5] J. Bradt et al., submitted to Nucl. Instrum. Methods Phys. Res. A (2017).

[6] D. Suzuki et al., Phys. Rev. C 87, 054301 (2013).

[7] A. Fritsch et al., Phys. Rev. C 93, 014321 (2016).

[8] F. Becchetti et al., Nucl. Instrum. Methods Phys. Res. A 505, 377 (2003).

[9] T. Suhara et al., Phys. Rev. C 82, 044301 (2010).

[10] H. L. Scott et al., Phys. Rev. 172, 1139 (1968).

[11] C. Force et al., Phys. Rev. Lett. 105, 102501 (2010).

[12] B. Bastin et al., Phys. Rev. Lett. 99, 022503 (2007).

[13] A. Gade et al., Phys. Rev. C 93, 054315 (2016).

[14] S. Calinescu et al., Phys. Rev. C 93, 044333 (2016).

[15] L. Gaudefroy et al., Phys. Rev. Lett. 97, 092501 (2006).

[16] J. J. Kolata et al., Nucl. Instrum. Methods Phys. Res. A 830, 82 (2016). 